\title{
Cudworth, Bayle y Hume: sobre el ateísmo estratonista
}

Fernando Bahr ${ }^{1}$

Resumen: Uno de los debates más interesantes en torno a la razonabilidad del materialismo ateo se dio entre los siglos XVII y XVIII a partir de que el anglicano Ralph Cudworth recuperó, para confutarla, una antigua versión atribuida al peripatético Estratón de Lampsaco. Esta versión, y las ideas de Cudworth al respecto, llegan al continente por obra de Jean Le Clerc, donde rápidamente caen bajo la crítica de Pierre Bayle. Bayle, en efecto, muestra que la posición de Cudworth era menos sólida de lo que se suponía y que, por lo tanto, la hipótesis estratonista no había sido confutada ni mucho menos. David Hume, por su parte, tomó nota de este debate y lo reutilizó como parte importante de sus Dialogues concerning Natural Religion, desde donde alcanzó su más amplia difusión.

Palabras clave: ateísmo - estratonismo - Cudworth - Bayle - Hume

\section{Cudworth, Bayle and Hume: on Stratonic atheism}

Abstract: One of the most interesting debates about the reasonableness of atheistic materialism occurred between the Seventeenth and Eighteenth Centuries after the Anglican Ralph Cudworth recovered, to confute it, an old version attributed to the peripatetic Strato of Lampsaco. This version, and Cudworth's ideas about it, reached the Continent through Jean Le Clerc, where they quickly fell under the criticism of Pierre Bayle. Bayle, in effect, shows that Cudworth's position was less solid than had been supposed and that, therefore, the Stratonic hypothesis had not been confuted at all. David Hume, for his part, took note of this debate and reused it as an important part of his Dialogues concerning Natural Religion, from where it was widely disseminated.

Keywords: atheism - Stratonism - Cudworth - Bayle - Hume

\footnotetext{
${ }^{1}$ Instituto de Filosofía "Ezequiel de Olaso" (INEO) - Consejo Nacional de Investigaciones Científicas y Técnicas-Centro de Investigaciones Filosóficas (CONICET-CIF) / Universidad Nacional del Litoral (UNL). E-mail: fernandobahr@gmail.com.
} 


\section{Cudworth, Le Clerc y Bayle}

En 1678, el platónico de Cambridge Ralph Cudworth (1617-1688) dio a conocer su opus magnum, The True Intellectual System of the Universe: Wherein all the Reason and Philosophy of Atheism is Confuted and its Impossibility Demonstrated. Esta obra, la única que Cudworth publicó en vida, es una impresionante muestra de erudición clásica de más de novecientas páginas infolio y puede ser leída como una historia de la filosofía antigua ${ }^{2}$. Cudworth la pensó como parte de un proyecto mayor cuyos objetivos eran: 1) demostrar la existencia de Dios, contra los ateos; 2) demostrar que ese Dios era esencialmente moral, bueno y justo, contra quienes lo concebían como "una omnipotente voluntad arbitraria que decreta, hace y vuelve necesarias todas las acciones, tanto malas como buenas"3; 3) demostrar la existencia de libertad o sui potestas en las criaturas racionales, contra quienes afirmaban que toda acción era intrínseca y esencialmente necesaria ${ }^{4}$. Murió diez años más tarde, en 1688, habiendo completado sólo la primera parte. Las otras dos iban a ser publicadas póstumamente a partir de numerosos manuscritos y notas que dejó ${ }^{5}$.

El libro se hizo célebre en la República de las Letras del siglo XVIII y su autor ha sido comparado con los principales filósofos de la época: Descartes, Leibniz, Malebranche. Esto puede ser probado a partir de un simple hecho: estando el philosophe ignorant de Voltaire en procura de entender algo acerca del universo y de sí mismo, uno de sus consejeros, después de Spinoza y de Leibniz, es Ralph Cudworth. Como en los otros casos, el pobre filósofo abandona a su maestro en el mismo estado de ignorancia que antes padecía, pero el dato es contundente. Para Voltaire, Cudworth formaba parte del canon de grandes filósofos, y su teoría metafísica estaba en pie de igualdad con la armonía preestablecida de Leibniz o con la sustancia de Spinoza 6 .

Como ha sugerido Luisa Simonutti, esta difusión del True Intellectual System en el continente se debió, al menos en parte, a una disputa que comenzó en Holanda a comienzos del siglo XVIII entre los refugiados hugonotes ${ }^{7}$. Los actores en esa disputa fueron, por una parte, el filólogo, filósofo y teólogo suizo Jean Le Clerc (1657-1736), y, por lo otra, el historiador y crítico francés Pierre Bayle (1647-1706). Le Clerc era editor de un journal de libros, noticias y reseñas llamado Bibliothèque choisie; allí, desde el primer volumen, empezó a publicar una traducción al francés -hecha por él mismo- de diversos pasajes del True Intellectual System. Estos largos pasajes - diez, en total ${ }^{8}$ - aparecieron en el curso de ocho volúmenes, a lo largo de tres años, y estuvieron acompañados por numerosos comentarios de elogio y algunas críticas. Las críticas tenían que ver, por ejemplo, con el valor que Cudworth concedía al argumento ontológico en la demostración de la existencia de Dios $^{9}$ o

\footnotetext{
2 John Locke la recomienda como tal. Cf. LOCKE, Some Thoughts Concerning Education, pp. 185-186

3 CUDWORTH, The True Intellectual System of the Universe, London, "Preface to the Reader". Salvo indicación en contrario, todas las traducciones son mías.

${ }^{4}$ CUDWORTH, The True Intellectual System of the Universe, London, "Preface to the Reader".

${ }^{5}$ La segunda parte en 1731 bajo el título A Treatise Concerning Eternal and Immutable Morality; la tercera en 1838, bajo el título $A$ Treatise of Free Will.

${ }^{6}$ Cf. VOLTAIRE, Le philosophe ignorant, p. 73.

${ }^{7}$ Cf. SIMONUTTI, "Bayle and Le Clerc as readers of Cudworth. Elements of the of the debate on Plastic Nature in the Dutch learned journals", p. 152.

${ }^{8}$ Véase LE CLERC, Bibliothèque choisie, I, pp. 63-138; II, pp. 11-77 y 78-130; III, pp. 11-106; V, pp. 30-145; VII, pp. 19-80; VIII, pp. 11-42 y 43-106; IX, pp. 1-40 y 41-103.

${ }^{9}$ Véase LE CLERC, Bibliothèque choisie, V, pp. 98, 133-134.
} 
a la teoría del innatismo ${ }^{10}$; los elogios, con la necesidad filosófica de encontrar un camino intermedio que evitara dos enemigos igualmente peligrosos: el ateísmo griego (y moderno), por una parte, $y$, por la otra, el ocasionalismo de los cartesianos radicalizados ${ }^{11}$.

De acuerdo con Cudworth, y Le Clerc, hay cuatro tipos de ateísmo: el de los "hylopatas" o seguidores de Anaximandro, el de los atomistas, el de los estoicos y el de los "hylozoicos"12. El último, el "hylozoico" o "estratonista" es el más peligroso, en tanto

adultera la noción de materia o cuerpo, mezclándola y confundiéndola con la de vida (...) y concluyendo de allí que a toda materia y sustancia como tal le pertenece esencialmente la vida y la percepción o entendimiento natural e inconsciente, y que en los animales el sentido y la razón o entendimiento conscientes nacen sólo por una modificación accidental en la organización de esta vida fundamental de la materia ${ }^{13}$.

Éste es uno de los peligros a evitar. Cudworth y Le Clerc consideran que ello es posible en tanto se demuestre que la materia no es nada más que masa resistente desprovista de vida, esto es, que el origen de la vida ha de ser exterior a la materia. Para evitar el peligro inverso, sin embargo, esa fuente de vida debe ser diferente de Dios, dado que en la suposición de que Dios haga "con sus propias manos, por decirlo así, cada mosca, cada polilla, cada larva y cada insecto" 14 , no habría un orden estable en la Creación y, peor aún, Dios mismo sería responsable de todos los desperfectos o fallos que se produjeran en ella.

Por lo tanto, dado que ni todas las cosas son producidas fortuitamente o por el mecanismo sin guía de la naturaleza, ni puede pensarse razonablemente que Dios las haga inmediata y milagrosamente, puede muy bien concluirse que hay una naturaleza plástica por debajo de Él, la cual, como un instrumento inferior y subordinado, se afana por ejecutar esa parte de la providencia que consiste en el movimiento regular y ordenado de la materia ${ }^{15}$.

La noción de "naturaleza plástica" es, como se sabe, el principal legado de Ralph Cudworth a la historia de la filosofía. En la escala boeciana del ser que Cudworth rehabilita ${ }^{16}$,

${ }^{10}$ Véase LE CLERC, Bibliothèque choisie, III, p. 32.

11 ROSA, "Ralph Cudworth in the République des Lettres: The Controversy about Plastick Nature and the Reputation of Pierre Bayle", p. 149. Por "cartesianos radicalizados" en este punto nos referimos a Louis de la Forge, Géraud de Cordemoy, Arnold Geulinx y, el más famoso de ellos, Nicolas Malebranche, quien en Recherche de la vérité había dicho: “il n'y a qu'un vraie cause, parce qu'il n'y a qu'un vrai Dieu (...) la nature ou la force de chaque chose n'est que la volonté de Dieu (...) toutes les causes naturelles ne sont point de véritables causes, mais seulement des causes occasionnelles" (MALEBRANCHE, Euvres complètes, II, p. 312).

12 "Il y a donc eu quatre sortes d'Atheïsme Philosophique. La premiere est celle des Hylopathiens, ou d'Anaximandre, dans laquelle on tire tout de la matiere destituée de sentiment, en lui attribuant des Formes et des Qualitez, qui s'y engendrent et qui s'y détruisent d'elles-mêmes. La seconde est celle des Atomistes, comme Democrite, qui fait tout venir du concours fortuit et de l'arrangement des Atomes. La troisiéme est l'Atheïsme Stoïcien, où une Nature aveugle, mais qui agit selon certaines regles, préside sur tout l'Univers. La quatriéme est l'Hylozoïque, ou celle de Straton, qui attribuoit à la matiere je ne sai quelle vie, sans sentiment et sans intelligence" (LE CLERC, Bibliothèque choisie, II, pp. 61-62).

${ }^{13}$ CUDWORTH, True Intellectual System, p. 144.

${ }^{14}$ CUDWORTH, True Intellectual System, p. 147 y LE CLERC, Bibliothèque choisie, II, p. 79.

${ }^{15}$ CUDWORTH, True Intellectual System, p. 150.

${ }^{16}$ Véase HUTTON, "Cudworth, Boethius, and the Scale of Nature”, pp. 93-100. 
esa naturaleza plástica es el tipo menos perfecto de sustancia incorpórea: más perfecta que la materia -mera masa resistente- es menos perfecta que los seres racionales o los animales en la medida en que le falta sentido (sense), conocimiento y conciencia ${ }^{17}$. Sus funciones incluyen la generación y conservación de plantas y animales ${ }^{18}$, o la regulación del movimiento de los cielos $^{19}$, pero su poder no es irresistible y, por lo tanto, "no completamente incapaz (de la misma manera que el arte humano) de verse algunas veces frustrado y decepcionado por la mala disposición de la materia"20.

Stefano Brogi presenta este último punto como la mejor explicación de los motivos que llevaron a Le Clerc a interesarse por la teoría de la "naturaleza plástica". El filólogo suizo encontró allí, dice Brogi, "un cómodo chivo expiatorio a cuya imperfección atribuir los males físicos del universo" 21 . Le Clerc, es cierto, vincula la metafísica de Cudworth con los avances en biología y sugiere, por ejemplo, que "el principio vital" de Nehemiah Grew (Cosmologia sacra, 1701) podría ser la confirmación empírica de esa metafísica; sin embargo, "no hay dudas de que el interés de Le Clerc por el vitalismo es de orden primariamente teológico y filosófico"22. Ahora bien, en ese sentido, ¿es la naturaleza plástica de Cudworth un chivo expiatorio eficaz, además de cómodo, para exculpar a Dios de los males del universo? Aquí entra a jugar el segundo actor en la contienda, Pierre Bayle.

El problema del mal tenía para Bayle un carácter recurrente, casi obsesivo: "una bidra -dice- a la que no se le puede cortar una cabeza sin que le renazcan las otras. Cuando usted piensa haberle tapado el camino a una objeción, sucede que ha abierto la puerta a varias otras dificultades"23. Así lo había mostrado en artículos como "Manichéens", "Pyrrhon" o "Pauliciens" de su Dictionnaire bistorique et critique ( $1^{a}$ edición: 1696; $2^{a}$ edición: 1702), donde declaraba a la teodicea cristiana vencida en argumentos cruciales (la doctrina del "permiso" del pecado, la relación entre libertad humana y providencia divina, la supuesta justicia del castigo eterno y el carácter inevitable de los males físicos) y derivaba de esa derrota una posición antirracional que denominaba -seria o sarcásticamente- "triunfo de la fe" y "llamado a la humildad cristiana" 24.

En las obras posteriores al Dictionnaire, y particularmente en la Continuation des pensées diverses (1704), Bayle insiste en estas consideraciones, pero acompañándolas de otras no menos inquietantes. Entre ellas, que las pruebas naturales de la existencia de Dios no son irrebatibles y que, por lo tanto, puede darse un ateísmo especulativo "de buena fe"25. Las reflexiones de Cudworth en The True Intellectual System respecto de los cuatro tipos de ateísmo

\footnotetext{
17 Véase ALLEN, pp. 337-347.

${ }^{18}$ Cf. CUDWORTH, True Intellectual System, pp. 151 y 167-168.

${ }^{19}$ Cf. CUDWORTH, True Intellectual System, p. 168.

${ }^{20}$ CUDWORTH, True Intellectual System, p. 150, y LE CLERC, Bibliothèque choisie, II, p. 84.

${ }^{21}$ BROGI, "Nature plastiche e disegni divini. La polemica tra Bayle e Le Clerc", pp. 55-56.

22 BROGI, "Nature plastiche e disegni divini. La polemica tra Bayle e Le Clerc", p. 54.

23 BAYLE, Réponse aux questions d'un provincial, (en adelante, RQP) en Euvres diverses (en adelante, OD), III, p. 631b.

24 "Hay tanta gente que examina tan poco la naturaleza de la fe divina, y que tan raramente reflexionan sobre este acto de su espíritu, que se hace necesario sacarlos de su indolencia mediante una larga lista de las dificultades que rodean los dogmas de la religión cristiana. Es por un vivo conocimiento de estas dificultades por lo que aprendemos la excelencia de la fe, y de este favor divino. Aprendemos por la misma vía la necesidad de desconfiar de la razón y de recurrir a la gracia. Aquellos que no hayan asistido jamás a los grandes combates de la razón y la fe y que ignoren la fuerza de las objeciones filosóficas, ignoran una buena parte del agradecimiento que le deben a Dios, y del método para triunfar sobre todas las tentaciones de la razón incrédula y orgullosa" (BAYLE, Dictionnaire bistorique et critique, IV, p. 646).

${ }^{25}$ BAYLE, Continuation des pensées diverses, (en adelante, CPD), en OD, III, p. 323b.
} 
en la Antigüedad le sirven justamente de prueba y abren la puerta a lo que será su último gran objeto de indagación: el naturalismo estratonista. Según éste, existe un orden eterno e inmutable que, sin origen en una causa inteligente anterior y sin estar dirigido por ningún conocimiento, puede ser considerado perfecto en sí mismo ${ }^{26}$. Bayle la considera en principio una hipótesis inadmisible. Pero igualmente inadmisibles a su juicio son todas las hipótesis que conciban a Dios de otra manera que como una voluntad absolutamente libre y creadora tanto de la existencia como de la esencia de las cosas, esto es, el arbitrarismo cartesiano. Desde el momento en que se acepten proposiciones de una verdad eterna que sean tales por naturaleza y no por una libre institución de Dios, verdades que Dios conoce necesariamente porque tal es su naturaleza, argumenta Bayle, "he allí una especie de fatum al cual Dios está sometido, he allí una necesidad natural absolutamente insuperable" ${ }^{27}$. Los estratonistas llaman "Dios" a una naturaleza que existe por sí misma sin haber sido hecha sobre ningún plan, idea o causa ejemplar anterior, y dan cuenta del orden al que tal naturaleza está sometida afirmando su propia necesidad, afirmando que es así y no de otra manera. Pero, ¿es que los teólogos pueden apelar a algún otro expediente a la hora de explicar, por ejemplo, por qué Dios no puede hacer lo que implica contradicción? ¿Qué ventajas supone demorar el encuentro con una primera causa cuya razón de ser no se explica por ningún plan anterior? ${ }^{28}$

Para Bayle, pues, lejos de explicarse como un resultado de la corrupción moral, el ateísmo estratonista es una posición filosófica coherente y difícil de refutar, tan difícil que incluso los que lo combaten con más ardor suelen darle armas sin darse cuenta. "El Sr. Cudworth y el Sr. Grew, grandísimos filósofos, son un ejemplo de ello" ${ }^{29}$. Estos autores, por evitar la hipótesis de una intervención directa y continua de Dios en la creación, y las ruinosas consecuencias que tal hipótesis tiene respecto de la teodicea, han atribuido a la naturaleza plástica o al principio vital la posibilidad de regular el universo sin tener conciencia de su función; con ello se han privado del único antídoto contra el ateísmo, a saber, el principio filosófico según el cual todo suceso que implica conformidad con leyes establecidas reenvía necesariamente a una causa inteligente que conoce de manera adecuada la ley general que es preciso respetar ${ }^{30}$. Los ateos infringen este principio adscribiendo la formación de animales a una causa que no es consciente de lo que hace y sigue un plan regular sin conocer la ley que ejecuta.

Pero la naturaleza plástica del Dr. Cudworth y el principio vital del Dr. Grew están exactamente en la misma situación, y, por lo tanto, su objeción contra los ateos es completamente ineficaz. Porque si Dios pudiera comunicar tal poder plástico, se seguiría que no es inconsistente con la naturaleza de las

\footnotetext{
26 " $[\mathrm{L}] \mathrm{a}$ naturaleza con sus virtudes y sus atributos, siendo el ser necesario, eterno e independiente, no puede encontrarse jamás bajo una forma que no emane de las leyes eternas e inmutables, y que en consecuencia no sea el estado más perfecto que pueda existir. No hay estado anárquico a temer en la máquina del mundo, ésta no puede estar más que en su estado natural, el caos o el retorno del caos no son más que quimeras; y si llegara un ordenamiento de los cuerpos distintos del que vemos, y que pareciera menos bello o más incómodo para el hombre, no sería por ello menos bello o más incómodo para todo el Universo" (BAYLE, CPD, en OD, III, p. 339b).

${ }^{27}$ BAYLE, $C P D$, en $O D$, p. 348a.

${ }^{28}$ Cf. BAYLE, CPD, en OD, III, p. 342a.

${ }^{29}$ BAYLE, CPD, en OD, pp. 216-217.

${ }^{30}$ Sobre la importancia de este principio en Bayle, puede verse MORI, Bayle philosophe, especialmente capítulos 3 y 5 .
} 
cosas que haya esos agentes. Podrían por lo tanto existir por sí mismos, diría el adversario, de lo cual también se seguiría que la regularidad que observamos en el universo podría ser el efecto de una causa ciega, que no fue consciente de lo que hizo ${ }^{31}$.

Observamos así que, según Bayle, el primer propósito de la obra de Cudworth, a saber, la refutación del ateísmo, no ha sido cumplido. Al contrario, los ateos, por lo menos en su versión estratonista, salen fortalecidos de la discusión. La única salida, por otra parte, parece seguir siendo la que Cudworth y Le Clerc querían evitar, esto es, la de un Dios que sin apoyarse en orden previo alguno -ni siquiera un orden de verdades- interviene directamente en el universo, creando y conservando a cada instante cada uno de los seres. Esta salida, sin embargo, como Bayle sabe, es desastrosa para cualquier proyecto de teodicea en tanto a nadie sino a Dios puede hacérsele responsable de los males de Su obra.

Ante un panorama tan desalentador, Le Clerc intentará repensar el concepto de "naturaleza plástica" comparándola con los pájaros que hacen su nido "de diversas maneras y compuesto de diversos materiales sin tener idea alguna de arquitectura, ni de orden, ni de albañilería", o con los artesanos que hacen una máquina sin saber el designio y las razones de aquel que la inventó ${ }^{32}$. Sin embargo, terminará por reconocer que no le es posible explicar cómo Dios dirige las acciones de sus criaturas sin ser el verdadero autor de ellas:

Confieso que no puedo decir cómo Dios utiliza la materia y dirige las naturalezas formatrices inmmateriales sin ser el autor de todas sus acciones, pero no se puede rechazar este pensamiento como absurdo después de las pruebas directas que he referido. De otra manera, habría que rechazar todo aquello de lo cual no tenemos ideas completas y exactas, lo cual nos haría caer en el más ridículo pirronismo ${ }^{33}$.

Era la confesión de una derrota. Le Clerc ha comprendido por fin, concluye Bayle por su parte, "1) que la carencia de toda idea no puede ser reemplazada en las naturalezas plásticas más que por una dirección de Dios; 2) que es imposible concebir que, bajo tal dirección, ellas conserven la cualidad de causa eficiente" ${ }^{34}$. No siendo la naturaleza plástica causa eficiente, la teodicea cudworthiana fracasa y toda la responsabilidad recae otra vez sobre la única causa verdadera, a saber, Dios mismo; un Dios que, insistimos, debe ser concebido a la manera de "Mr. Descartes y una parte de sus seguidores": como causa libre de las verdades y de las esencias y, en tal sentido, como pudiendo hacer un círculo cuadrado

\footnotetext{
${ }^{31}$ BAYLE, CPD, en OD, III, p. 217a. "La doctrina de Mr. Cudworth consiste en: 1) Que la verdadera causa próxima e inmediata de los animales no tiene ninguna idea de la obra que produce. 2) Que ella ha sido hecha por una causa que sí tiene idea de esa obra. Los estratonistas admiten la primera de estas dos proposiciones y rechazan la segunda. Por poca sinceridad que tengan, reconocerán que no hay nada más extraño ni más incomprensible que suponer que lo que forma la máquina de los animales no conozca nada, y sin embargo logre poner cada parte en el lugar que corresponda para la construcción de una obra donde se encuentra más artificio que en todo lo que el género humano ha inventado de mayor maravilla e ingenio. Pero sostendrían que Mr. Cudworth no puede molestarlos con esto, porque ya la primera proposición de su doctrina contiene este gran inconveniente, y su segunda tesis no lo puede remediar" (BAYLE, RQP, en OD, III, p. 888 a-b).

${ }^{32}$ LE CLERC, Bibliothèque choisie, VII, pp. 282-283.

${ }^{33}$ LE CLERC, Bibliothèque choisie, VII p. 287.

${ }^{34}$ BAYLE, RQP, en OD, III, p. 890b.
} 
si se le ocurriera ${ }^{35}$. Tal es para Bayle la única muralla que la filosofía cristiana puede oponer al estratonismo.

¿Suscribía el mismo Bayle esa teoría del arbitrarismo divino? Dicho de otra manera, una vez admitido que no había paso intermedio como pretendían Cudworth y Le Clerc, ¿prefería Bayle el Caribdis del cartesianismo radicalizado para evitar la Escila del até́smo hylozoico o estratonista? Ciertas declaraciones nos llevarían a pensar que sí. En efecto, Bayle suele mostrarse confiado en la teoría cartesiana ("todas estas dificultades se evaporan desde que suponemos que las esencias de las criaturas y las verdades filosóficas han sido fijadas mediante actos de la voluntad de Dios" ${ }^{36}$ ) y califica en cambio de "incomprensible" la hipótesis estratonista. Pues ésta obligaría a quienes la defienden "a decir que una naturaleza que no tiene ni vida ni sentimiento, ha producido todas estas bellas obras, y que, sin saber lo que hacía les ha dado una simetría y una subordinación que parecen evidentemente el efecto de una inteligencia muy esclarecida, y que elige sus medios y sus fines" ${ }^{\prime 3}$. Por otro lado, no deja de reconocer que las consecuencias últimas del cartesianismo son terribles. Entre otras cosas, porque elimina cualquier distinción natural entre el vicio y la virtud ${ }^{38}, \mathrm{y}$, por dar lugar “a la pretensión de que esta proposición, tres y tres son seis, no es verdadera más que en el lugar y durante el tiempo que Dios lo quiera; que podía ser muy falsa en algunas partes del Universo, y que acaso lo será entre los hombres el año que viene" ${ }^{39}$, nos conduce al pirronismo más exagerado. Se les puede endilgar, por lo tanto, a los estratonistas que su teoría es insostenible racionalmente, pero ¿no podrían ellos acaso replicar que la que aparentemente los refuta también lo es? Se les puede endilgar que terminan afirmando algo incomprensible, pero ¿no podrían ellos replicar exactamente lo mismo? En este atolladero nos deja Bayle.

\section{Los Dialogues de Hume}

Se conocen bajo el nombre de "early memoranda" un conjunto de 318 notas de lectura tomadas por David Hume en veintiséis folios carentes de numeración original y distribuidas en tres secciones.$^{40}$ La primera sección, denominada por su autor "Filosofía natural", es sólo un folio con nueve notas; la segunda sección, "Filosofía", abarca cuarenta notas y cinco folios. Los veinte folios restantes, sin título, contienen 269 notas de contenido variado y han sido agrupados en una sección bajo el rótulo de "General".

\footnotetext{
${ }^{35}$ Cf. BAYLE, CPD, en OD, III, p. 348a.

36 BAYLE, $C P D$, en $O D$, III, p. 348a.

${ }^{37}$ BAYLE, $C P D$, en $O D$, III, p. 333a.

38 "La consecuencia de esta doctrina sería que, antes de que Dios se determinara a crear el mundo, no veía nada mejor en la virtud que en el vicio, y que sus ideas no le mostraban que la virtud fuera más digna de su amor que el vicio. Esto elimina toda distinción entre el derecho natural y el derecho positivo; no habría ya nada inmóvil o indispensable en la moral, habría sido igualmente posible para Dios ordenarnos ser viciosos que ordenarnos ser virtuosos, y jamás podríamos estar seguros de que las leyes morales no serían un día abrogadas, como lo han sido las leyes ceremoniales de los judíos." (BAYLE, RQP, en OD, III, p. 675b). Esta teoría cartesiana, por otra parte, volvía inexplicable una idea que Bayle deseaba defender: la de que los ateos, siguiendo su razón, pudieran actuar moralmente, es decir, pudieran reconocer una deshonestidad intrínseca en el vicio y una honestidad intrínseca en la virtud, Cf. BAYLE, CPD, en OD, III, pp. 408b-410b.

${ }^{39}$ BAYLE, RQP, en OD, III, pp. 675b-676a.

40 Nos atenemos a los datos aportados en la edición de MOSSNER, "Hume's Early Memoranda, 1729-1740: The Complete Text”, pp. 492-518.
} 
La que nos interesa aquí es la sección "Filosofía", pues en ella se tratan temas relacionados con cuestiones de teología natural, temas que serán objeto de análisis en escritos posteriores de Hume, sobre todo en los póstumos Dialogues concerning Natural Religion (1779). En ella, además, se nombra o se alude a Pierre Bayle con frecuencia; concretamente, veinticuatro de las cuarenta notas, como hemos mostrado en otro trabajo ${ }^{41}$, han sido tomadas, con seguridad, de Bayle; más precisamente, del volumen III de sus Oeuvres diverses: dos de Pensées diverses sur la comète, siete de la Continuation des pensées diverses y quince de la Réponse aux questions d'un provincial.

La hipótesis estratonista está presente entre ellas, por supuesto. En la nota 15, por ejemplo, que reza así:

Un estratonista podría replicar los argumentos de todas las sectas filosóficas. De los estoicos, que mantuvieron que su Dios era ardiente y compuesto, y de los platónicos que afirmaron que las ideas eran distintas de la Divinidad. La misma pregunta ¿por qué las partes o las ideas de Dios tienen esta disposición particular? es tan difícil como por qué tiene el mundo esta disposición particular.

No lo nombra a Bayle, pero proviene sin dudas del capítulo CVI de la Continuation des pensées diverses. Intentaremos a continuación ver cómo reaparece en los Dialogues concerning Natural Religion y cuál es el contexto de esa reaparición.

Vayamos, pues, a los Dialogues. En ellos se relata una conversación entre tres hombres de letras -Cleanthes, Philo y Demea- tal como ha quedado grabada en la memoria de un auditor más joven, de nombre Pamphilus, discípulo de Cleanthes. El tema que reúne a los tres amigos es el modo más adecuado de inculcar la piedad religiosa en los alumnos o hijos. Se trata de un asunto pedagógico, por lo tanto; sin embargo, deriva en un largo debate acerca de la posibilidad de apoyar las creencias teológicas en argumentos racionales. Philo niega tal posibilidad; Demea confía de manera exclusiva en el tradicional argumento a priori; Cleanthes, finalmente, el más firme defensor de un aval racional para la fe, va a desarrollar el llamado "argumento del diseño" (the design argument) con vistas a sostener su posición.

Este argumento es, como se sabe, de tipo analógico, es decir, un argumento que parte de la semejanza aparente entre los efectos para inferir una semejanza en las causas. En el caso que nos ocupa, los efectos a comparar son, por un lado, la coherencia de partes y la adaptación de medios a fines observables en la naturaleza y, por el otro, los productos "del diseño, el pensamiento, la sabiduría y la inteligencia humanas". Puesto que los efectos se asemejan, "nos sentimos inclinados a inferir, por todas las reglas de la analogía, que también las causas se asemejan, y que el Autor de la naturaleza es en algo similar a la mente del hombre, aunque dotado de facultades mucho más amplias, en proporción con la grandeza de la obra que ha ejecutado" ${ }^{42}$. De esta manera, dice Cleanthes, y sólo de esta manera, se

\footnotetext{
${ }^{41}$ Para un análisis más detallado, véase BAHR, "Pierre Bayle en los early memoranda de Hume", pp. 7-38. También KREIMENDAHL, "Die Kirche ist mir ein Greuel”. Studien zur Religionsphilosophie David Humes, cap. IXXI. Para una perspectiva más amplia sobre la influencia de Bayle en los Dialogues de Hume, son imprescindibles: PAGANINI, "Theism, Atheism, and Scepticism. Bayle’s Background to Hume's Dialogues", pp. 203-243, y PAGANINI, "Hume's 'questions of words' and Bayle's 'disputes de mot'. On Dialogues XII", pp. 51-71.

${ }^{42}$ HUME, Dialogues concerning Natural Religion (de aquí en adelante, DNR), ed. Gaskin, II, p. 45.
} 
prueba al mismo tiempo la existencia de una deidad y la semejanza de esa deidad con la mente y la inteligencia humanas.

Apenas Cleanthes concluye su alegato, Philo identifica un supuesto en el argumento, supuesto que posteriormente se convertirá en una grave objeción. A priori, dice Philo, es posible suponer que la materia contiene dentro de sí el origen del orden, de la misma manera que lo contiene la mente, pues "no es más difícil concebir que los diversos elementos pueden disponerse, por una desconocida causa interna, en la más exquisita de las ordenaciones, que concebir que sus ideas adoptan la misma ordenación por una desconocida causa interna semejante en la gran mente universal" ${ }^{\prime 3}$. A priori, pues, el orden o el ajuste de las causas finales no son por sí mismos prueba alguna de diseño. El argumento de Cleanthes se apoya, sin embargo, en que la experiencia nos indica que las ideas en la mente humana, "gracias a una desconocida e inexplicable economía", se organizan por sí mismas para concebir el plan de un artefacto, mientras que si nos limitamos a amontonar piezas metálicas ese artefacto no se produce por su propia cuenta. La experiencia, por lo tanto, parece enseñarnos que la mente cuenta con un principio de orden interno, y que tal principio no existe en la materia. A partir de allí, concluye Philo, Cleanthes estaría en condiciones de inferir que el universo, al igual que los artefactos inventados por el hombre, carece de un principio de orden interno y que tal principio reside en una mente original que lo ha construido: "de efectos similares inferimos causas similares" $"$.

Philo declara en aquel momento aceptar dicho supuesto a los fines de la discusión. En la Parte IV, sin embargo, el "experimento crucial" de Cleanthes comienza a ser puesto en cuestión. Un mundo mental o universo de ideas, dice Philo, requiere de una causa tanto como un mundo material o universo de objetos. Así, pues, poco se gana con explicar el mundo material a partir de un mundo mental anterior. Se ha dado un paso, pero puede ser un paso al infinito. Ese mundo mental, acaso, ¿no deberá ser explicado a partir de otro mundo mental anterior o de un nuevo principio inteligente? ¿Y este nuevo principio no necesitaría de otro anterior, y éste de otro, sin término final? Nada hay más insatisfactorio que la progresión ad infinitum.

Sería mejor, por lo tanto, no asomarse nunca más allá del presente mundo material. Al suponer que éste contiene en sí mismo el principio de su ordenamiento, afirmamos realmente que es Dios; y tanto mejor cuanto más pronto lleguemos a ese Ser divino. Cualquier paso que te conduzca más allá del sistema mundano sólo despertará en ti una disposición inquisitiva a la que jamás lograrás satisfacer ${ }^{45}$.

El argumento del diseño se enfrenta aquí a su máximo adversario: la hipótesis naturalista o "estratonista" a la que aludía Hume en los memoranda y que ya habían tratado, como vimos, Cudworth, Le Clerc y Bayle. Dijimos que esta nota en concreto provenía del capítulo CVI de la Continuation des pensées diverses. Allí, en efecto, Bayle imagina la réplica que un discípulo de Estratón podría haber dirigido a los estoicos o a los platónicos que criticaran el supuesto naturalista de un mundo que, sin haber sido hecho por una causa inteligente

${ }^{43}$ HUME, DNR, II, p. 48.

${ }^{44}$ HUME, DNR II, p. 48.

${ }^{45}$ HUME, DNR, IV, p. 64. 
anterior, tuviera un orden en sí mismo. Tal réplica consiste en mostrar que si es difícil explicar cómo hay un orden en la materia sin la dirección de un ser inteligente, no es menos difícil explicar cómo hay un orden en Dios sin la dirección de un ser inteligente. En este sentido, justamente, camina el razonamiento de Philo. "Decir que las diferentes ideas que componen la razón del Ser supremo se ordenan por sí mismas y por su propia naturaleza, es realmente hablar sin un sentido preciso. Y si tiene un sentido, me gustaría saber por qué no lo tiene asimismo decir que las partes del mundo material se ordenan por sí mismas y por su propia naturaleza"46. Las dos proposiciones son igualmente inteligibles o igualmente ininteligibles. Y la segunda cuenta con el principio de economía a su favor: no multiplicar innecesariamente las entidades.

Pero hay algo más. Los estratonistas apelaban a la condición de causa primera de su naturaleza para negar validez a las preguntas acerca de por qué era de una manera y no de otra. "La naturaleza es asi", tal era toda su respuesta. Pierre Bayle acepta que esa estrategia puede ser discutible; sin embargo, admite también que tarde o temprano cualquier otra hipótesis debe concluir en una afirmación similar: detenerse en el establecimiento de la naturaleza del primer ser, sin poder buscar la razón de sus atributos en un orden o en un plan anterior ${ }^{47}$. Philo, por su parte, compara esta dificultad, y la "solución" a ella, con la apelación que los peripatéticos hacían a las "facultades o cualidades ocultas" cuando no querían conceder que desconocían la causa de un fenómeno. Vosotros los antropomorfistas, le dice a Cleanthes, cuando se os pregunta por la causa que produce orden en las ideas del Ser supremo, sólo decís que se trata de una facultad racional y que tal es la naturaleza de la deidad. ¿Por qué no sería válida una respuesta similar para explicar el orden del mundo sin tener que recurrir a un creador inteligente?

Es sólo cuestión de decir que tal es la naturaleza de los objetos materiales, y que todos ellos poseen originalmente una facultad de orden y proporción. Éstas no son sino maneras más cultas y elaboradas de confesar nuestra ignorancia; y la primera hipótesis no tiene ninguna ventaja real sobre la otra, excepto en guardar más conformidad con los prejuicios del vulgo ${ }^{48}$.

Las afinidades con Pierre Bayle parecen no terminar allí. En la Parte VI, Philo expone de una manera más enfática la necesidad de recurrir tarde o temprano al hecho último de un principio eterno de orden para dar cuenta de la regularidad natural y evitar el abismo de un regreso infinito. El pasaje ha suscitado varios comentarios, y, de hecho, no deja de presentar algunos puntos oscuros ${ }^{49}$. Dice Philo:

Y si me viera obligado a defender un sistema de esta naturaleza [es decir, de acuerdo con el principio empírico "lo que vemos en las partes podemos inferirlo del todo’], lo que de buena gana jamás haría, estimaría que ninguno

\footnotetext{
${ }^{46}$ HUME, DNR, IV, p. 64.

${ }^{47}$ Cf. BAYLE, CPD, en OD, III, p. 342a.

${ }^{48}$ HUME, DNR, IV, p. 65.

$49 \mathrm{Al}$ respecto, puede verse: PYLE, Hume's Dialogues concerning Natural Religion. A Reader's Guide, pp. 70-71; TWEYMAN, Scepticism and Belief in Hume's, pp. 81-83; GASKIN, Hume's Philosophy of Religion, pp. 35-36; y NATHAN, “Hume’s Immanent God”, pp. 412-414.
} 
es más plausible que el que adscribe al mundo un eterno e inherente principio de orden, aunque escoltado por grandes y continuas revoluciones y alteraciones ${ }^{50}$.

El tono inusualmente afirmativo de la última parte no puede dejar de sorprender en un escéptico. Philo estaría proponiendo aquí que suponer al mundo (a la materia, deberíamos entender) como dotado de un principio de orden "eterno e inherente" resulta la hipótesis cosmológica más verosimil. Con lo cual, al parecer, no sólo estaría objetando el argumento del diseño sino también sosteniendo la hipótesis estratonista o naturalista. Contra esta interpretación, es cierto, se puede alegar la restricción impuesta al comienzo parafraseándola: "jamás intentaría de buena gana defender un sistema de la naturaleza a partir del principio empírico lo que vemos en las partes podemos inferirlo del todo". De todas maneras, puesto ante el dilema, Philo acepta que el estratonismo le resulta más fácil de defender, y por ello más plausible, que la hipótesis religiosa.

Desde aquí es posible establecer, decíamos, un nuevo vínculo entre Hume y Bayle. En efecto, en la Continuation des pensées diverses este último se pregunta si resultaba posible para los filósofos paganos - esto es, para la pura razón, sin ayuda revelada- remontarse desde los efectos visibles en el mundo hacia la existencia de Dios, es decir, hacia la existencia de una naturaleza "que, sin extensión alguna, y sin composición alguna, tiene una ciencia, una sabiduría, una bondad, una justicia, una potencia infinitas" ${ }^{51}$. Su respuesta es negativa. Nadie que supusiera la eternidad de la materia -como se debe hacer, de acuerdo a razones estrictamente filosóficas- y que pensara en un Dios realmente extenso -como se debería hacer, si se quiere explicar el poder de la divinidad para mover los cuerpos- puede llegar a concebir la naturaleza perfecta. Esto es, el primer ser que "por un acto único de su entendimiento conoce todas las cosas, y que por un acto único de su voluntad mantiene un cierto orden en el universo o lo cambia según su capricho" 52 .

Retengamos esta última condición. Un primer ser que por un acto único de su voluntad mantiene un cierto orden en el universo o lo cambia según su capricho. Tal era la hipótesis de los cartesianos, la hipótesis "arbitrarista", y Bayle, como vimos, considera que es la única manera de enfrentar a los estratonistas: sostener "como Mr. Descartes y una parte de sus seguidores, que Dios es la causa libre de las verdades, y que él podría hacer un círculo cuadrado cuando quisiera" ${ }^{53}$.

Hume conocía bien este problema. Lo atestigua la nota de los Memoranda inmediatamente anterior a la que citamos al comienzo de esta sección, la número 14: "El ateísmo de Estratón, el más peligroso de los antiguos, sostenía que el origen del mundo era la naturaleza o una materia dotada de actividad. Bayle piensa que sólo los cartesianos pueden refutar este ateísmo." Ahora bien, hemos visto que el asunto es más complejo, pues la "refutación" que Bayle tenía en mente dependía de que se aceptaran condiciones sin fundamento racional. Estrictamente, pues, no hay refutación; lo único que puede hacerse

\footnotetext{
${ }^{50}$ HUME, DNR, VI, p. 76. Gaskin hace notar que Hume había escrito inicialmente "in matter" ("en la materia") en lugar de "to the world" ("al mundo").

${ }^{51}$ BAYLE, CPD, en OD, III, p. 330a.

52 "De ahí la esperanza de sabernos escuchados cuando le rezamos; el miedo a ser castigados cuando nos conducimos mal; la confianza de vernos recompensados cuando vivimos bien; toda la religión, en una palabra, y sin ello ninguna religión" (BAYLE, CPD, en OD, III, p. 329b).

${ }^{53}$ BAYLE, CPD, en OD, III, p. 348a.
} 
respecto de los estratonistas es desconcertarlos, oponiéndoles la hipótesis cartesiana y haciéndoles ver que ésta escapa a su temible réplica porque -a diferencia de los platónicos, por ejemplo- no supone orden alguno, excepto el que momentáneamente surja de la voluntad de Dios.

Cuál fue la palabra final de Bayle en esta controversia y, por ende, en qué medida él mismo podría ser considerado un defensor del estratonismo, un ateo, es, lo mencionamos antes, una cuestión muy debatida. Lo que sí podría decirse con cierta seguridad es que, en cuanto posición filosófica, consideraba el estratonismo una hipótesis muy difícil de vencer. No sabemos si esto, que Bayle sostuvo hacia 1704, podría tener alguna relación con lo que Hume hizo decir a su personaje escéptico, Philo, varias décadas más tarde, a saber, que si se viera obligado a defender un sistema de acuerdo con el método analógico, estimaría que ninguno es más plausible que el que adscribe al mundo un eterno e inherente principio de orden. En todo caso, renovando una sugerencia ya hecha por Norman Kemp Smith en $1948^{54}$, las semejanzas son ciertas y las notas 14 y 15 de los early memoranda nos autorizan a sostenerlas.

\section{Conclusiones}

En The True Intellectual System of the Universe, Ralph Cudworth lleva a cabo una reconstrucción del pensamiento antiguo buscando principalmente arrancar de raíz la posibilidad del ateísmo y defender la teoría teológica cristiana. En el curso de esa reconstrucción recupera una variante del ateísmo griego que hasta entonces había pasado casi desapercibida: el ateísmo hylozoico o estratonista, vinculado a un oscuro filósofo peripatético: Estratón de Lampsaco.

Jean Le Clerc, entusiasmado por el proyecto apologético de Cudworth, toma su clasificación de los ateísmos antiguos. Para apoyar y difundir a Cudworth, traduce -en la Bibiothèque choisie, journal que dirigía- muchos pasajes del True Intellectual System al francés. Allí lo leyó Pierre Bayle, quien no conocía el inglés, y tal lectura significó el principio del fin de la empresa cudworthiana.

En efecto, discutiendo con Le Clerc, Bayle muestra que la supuesta refutación del ateísmo era inviable y que la hipótesis de la "naturaleza plástica", lejos de salvar a Dios de los males del mundo, sólo es un subterfugio que no disminuye en nada Su responsabilidad. Después de Bayle, por lo tanto, el dilema es de hierro: o adoptamos el arbitrarismo cartesiano, con todas sus horribles consecuencias, o aceptamos que la hipótesis estratonista es irrefutable.

Para terminar. Varios de los pasajes en que Bayle discute con Le Clerc y, por su intermedio, con Cudworth, fueron incorporados por David Hume a los "early memoranda", en notas que constituyen parte de la materia prima con que el filósofo escocés compuso posteriormente los Dialogues concerning Natural Religion. De hecho, el personaje escéptico de los Dialogues, Philo, trata la hipótesis estratonista en términos muy semejantes a los de Bayle $\mathrm{y}$, como Bayle, termina defendiéndola $-\mathrm{O}$, al menos, aceptándola- por un principio de economía. Se produce así una vuelta del estratonismo desde el continente a las islas, vuelta

${ }^{54}$ HUME, Dialogues concerning Natural Religion, p. 36. 
que parece darle más motivos al temor de Cudworth por esa hipótesis a la que consideraba "el sol naciente del ateísmo" "55.

\section{Referencias bibliográficas}

ALLEN, Keith. "Cudworth on Mind, Body, and Plastic Nature". In: Philosophy Compass, 8/4, 2013, pp. 337-347.

BAHR, Fernando. "Pierre Bayle en los early memoranda de Hume". In: Revista Latinoamericana de Filosofía, Vol. XXV, No 1, Otoño 1999, pp. 7-38.

BAYLE, Pierre. Dictionnaire historique et critique. $5^{a}$ ed., Amsterdam/Leyde/La Haye/Utrecht, P. Brunel et al., 1740.

Réponse aux questions d'un provincial (RQP), en CEuvres diverses (OD). III, La Haye, P. Husson et al., 1727-1731.

Continuation des pensées diverses (CPD), CEuvres diverses (OD). III, La Haye, P. Husson et al., 1727-1731.

BROGI, Stefano. "Nature plastiche e disegni divini. La polemica tra Bayle e Le Clerc". In: Studi Settecenteschi, 20, 2000.

CUDWORTH, Ralph. The True Intellectual System of the Universe: Wherein all the Reason and Philosophy of Atheism is Confuted and its Impossibility Demonstrated. London: printed for Richard Royston, 1678.

. A Treatise Concerning Eternal and Immutable Morality, With a Treatise of Freewill. Edited by Sarah Hutton. Cambridge: Cambridge UP, 1996.

GASKIN, J. C. A. Hume's Philosophy of Religion. London and Basingstoke: The MacMillan Press, 1978.

HUME, David. Dialogues concerning Natural Religion (DNR). In : Dialogues and Natural History of Religion. Edited by J. C. A Gaskin. Oxford/New York: Oxford University Press, 1993.

Dialogues concerning Natural Religion. Edited with an introduction by Norman Kemp Smith, second edition, with supplement. New York: Social Sciences Publishers, 1948.

HUTTON, Sarah. "Cudworth, Boethius, and the Scale of Nature". In: The Cambridge Platonists in Philosophical Context: politics, metaphysics, and religion. Rogers, Vienne y Zarka (eds.). Dordrecht: Springer, 1997.

KREIMENDAHL, Lothar. "Die Kirche ist mir ein Greuel". Studien zur Religionsphilosophie David Humes. Würzburg: Königshausen und Neumann, 2012.

55 “[T]he Rising Sun of Atheism” (CUDWORTH, True Intellectual System, p. 145). 
LE CLERC. Bibliothèque choisie. Amsterdam: Henri Schelte, 1703-1713.

LOCKE, John. "Some Thoughts Concerning Education". In: The Works of John Locke in Nine Volumes, 12th. edition, Vol. 8, London, 1824.

MALEBRANCHE, Nicolas. Oeuvres complètes. Éd. André Robinet. Paris : Vrin, 1974.

MORI, Gianluca. Bayle philosophe. Paris: Honoré Champion, 1999.

MOSSNER, Ernest C. “Hume's Early Memoranda, 1729-1740: The Complete Text”. In: Journal of the History of Ideas, Volume IX, No 4, October 1948, pp. 492-518.

NATHAN, George. “Hume’s Immanent God”. In: Hume. V. C. Chappell (ed.). New York: Anchor Books, 1966.

PAGANINI, Gianni. “Theism, Atheism, and Scepticism. Bayle's Background to Hume's Dialogues". In: W. Schröder (éd.), Gestalten des Deismus in Europa. Wiesbaden: Harrassowitz Verlag, 2013, pp. 203-243.

PAGANINI, Gianni. 'Hume's 'questions of words' and Bayle's 'disputes de mots'. On Dialogues XII". In: Hume et la religion. Nouvelles perspectives, nouveaux enjeux. S. Charles (ed). Hildesheim: Olms, 2013.

PYLE, Andrew. Hume's Dialogues concerning Natural Religion. A Reader's Guide. London/New York: Continuum, 2006, pp. 70-71

ROSA, Susan. "Ralph Cudworth in the République des Lettres: The Controversy about Plastick Nature and the Reputation of Pierre Bayle". In: Studies in Eighteenth Century Culture, Vol. 23, Johns Hopkins University Press, 1994.

SIMONUTTI, Luisa. "Bayle and Le Clerc as readers of Cudworth. Elements of the of the debate on Plastic Nature in the Dutch learned journals". In: Geschiedenis van de Wijsbegeerte in Nederland, 4, 2, 1993.

TWEYMAN, Stanley. Scepticism and Belief in Hume's Dialogues. Dordrecht/Boston/Lancaster: Martinus Nijhoff, 1986.

VOLTAIRE. "Le philosophe ignorant". In: CEuvres complètes de Voltaire, texte établi par Louis Moland, Garnier, tome 26. Paris: Garnier, (1766), pp. 47-96. 